\title{
Benign Miliary Osteoma Cutis of the Face: A Common Incidental CT Finding
}

\author{
(DD. Kim, DG.A. Franco, DH. Shigehara, (D). Asaumi, and (D). Hildenbrand
}

\begin{abstract}
BACKGROUND AND PURPOSE: Osteoma cutis of the face represents a primary or secondary formation of ossific foci in the facial skin. Its primary form has been sparsely described in the plastic surgery and dermatology literature. As radiologists, we routinely encounter incidental, very small facial calcified nodules on CT studies performed for a variety of unrelated reasons. We hypothesized that this routinely encountered facial calcification represents primary miliary osteoma cutis and is a common, benign, age-related finding.
\end{abstract}

MATERIALS AND METHODS: We retrospectively reviewed 1315 consecutive sinus CTs obtained during an 8-month period and their associated demographics. The number of dermal radiopaque lesions with Hounsfield units of $>150$ was counted, and we analyzed the association between the prevalence of these lesions and patients' demographics with logistic regression methods.

RESULTS: Five hundred ninety-nine males and 716 females from 4 to 90 years of age were included in the study (mean, 52 versus 51 years; $P=.259)$. Among these, 252 males and 301 females had small facial calcified nodules ( $42.1 \%$ versus $42.0 \%, P=.971)$. The patient's age was a statistically significant predictor for having facial calcified nodules (odds ratio $=1.02, P<.001$ ), while the patient's sex was not $(P=.826$ ).

CONCLUSIONS: Facial calcified nodules, observed in routine head and face CT imaging, are common, benign, age-related findings, which have been largely overlooked in the radiology literature. It is a manifestation of primary miliary osteoma cutis.

0 steoma cutis (cutaneous ossification) represents primary or secondary formation of ossific foci in the skin. It was first described by Martin Wilckens in $1858 .{ }^{1}$ It is distinguished radiographically and pathologically from calcinosis cutis by the deposition of organized bone matrix, while the latter is characterized by the deposition of amorphous calcium salts within the skin. ${ }^{2-4}$ Some consider calcinosis cutis a precursor or the early manifestation of osteoma cutis. ${ }^{5}$ Similar to secondary cutaneous calcification, the etiologies of secondary osteoma cutis have been welldescribed in the radiology and dermatopathology literature and are attributed to iatrogenic, traumatic, metabolic (eg, Albright hereditary osteodystrophy), inflammatory (eg, acne or dermatomyositis), and neoplastic (eg, basal cell carcinoma) processes. ${ }^{2,5-7}$

Received September 6, 2016; accepted after revision December 6.

From the Department of Radiology (D.K., P.H.), Lahey Hospital and Medical Center Burlington, Massachusetts; Commonwealth Radiology Associates (G.A.F.), Brock-

ton, Massachusetts; and Department of Oral and Maxillofacial Radiology (H.S., J.A.) Okayama University Graduate School of Medicine, Dentistry, and Pharmaceutical Sciences, Okayama, Japan.

Paper previously presented at: Annual Meeting of the American Society of Neuroradiology and the Foundation of the ASNR Symposium, May 23-26, 2016; Washington, DC.

Please address correspondence to Peter Hildenbrand, MD, Department of Radiology, Lahey Hospital and Medical Center, 41 Mall Rd, Burlington, MA 01805; e-mail:

Peter.Hildenbrand@Lahey.org

http://dx.doi.org/10.3174/ajnr.A5096
However, primary or idiopathic osteoma cutis has been sparsely described as a rare disease entity in the plastic surgery and dermatology literature, mostly in the form of case reports. ${ }^{4,6,8-11}$

The most frequently reported subclass of primary osteoma cutis occurs in the face and scalp soft tissues. It is reported as "miliary osteoma cutis of the face." ${ }^{\prime, 10,12,13}$ Current literature agrees on its idiopathic etiology and its benign, noninvasive, and asymptomatic nature. ${ }^{4,6,10,12,13}$ However, there have been varying reports regarding its epidemiology. While several authors describe it as a very rare disease $^{6,9,12}$ with slightly increased incidence in elderly or middle-aged women, ${ }^{2,14,15}$ other authors report it as a very common entity in the general population, ${ }^{16}$ without sex or age predilection. ${ }^{6}$

As radiologists, we routinely encounter incidental, punctate facial hypodermal calcifications on CT studies performed for a variety of reasons in patients without clinically recognized underlying dermatopathology (Fig 1). Due to their benign and asymptomatic nature, these incidental facial calcified nodules have been largely overlooked in the imaging literature. We performed a retrospective review of a large CT dataset. Our imaging technique extended the results of a previously reported large, radiographic, cadaveric case series, ${ }^{16}$ in an effort to establish that routinely encountered facial dermal calcification/ossification is primary miliary osteoma cutis, a common, benign, age-related finding. 


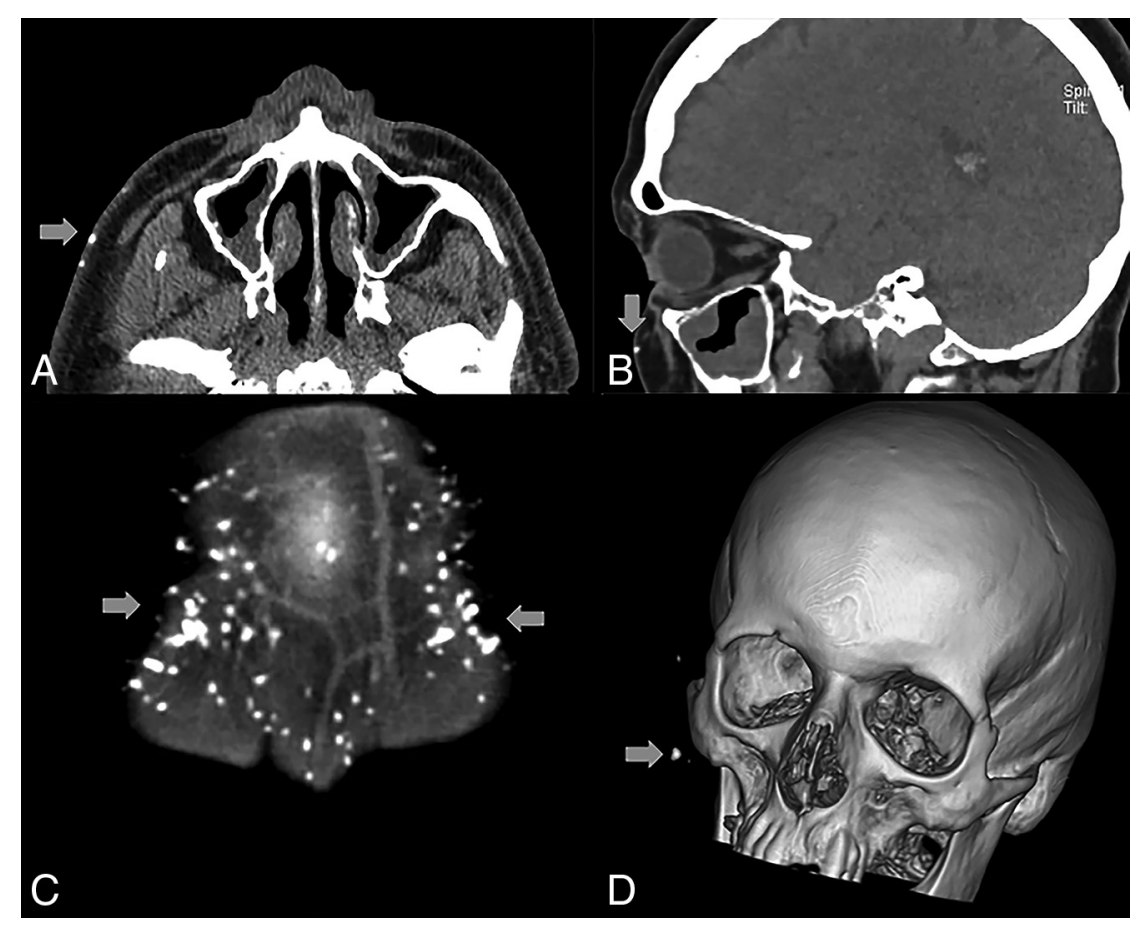

FIG 1. Axial (A), sagittal (B), and coronal (C) CT images of 3 different representative patients demonstrate multiple millimetric scattered facial and scalp hypodermal calcified nodules with varying degrees of severity. A 3D bone window reconstruction of a patient's sinus $C T(D)$ also demonstrates a relatively large, 4- to 5-mm facial calcified nodule within the right premaxillary skin.

titative effect of the subject's age and sex on the number of facial calcified nodules, we performed the Kruskal-Wallis test with post hoc analysis (Dunn test), with age decade and sex as qualitative independent variables. The KruskalWallis test was chosen given the nonparametric nature of our study population. The prevalence of calcified nodules and the mean ages between male and female sex were compared with the $\chi^{2}$ and $Z$-tests, respectively. All statistical calculation was performed with MedCalc for Windows software (MedCalc Software, Mariakerke, Belgium). $P<.05$ was considered a cutoff value for statistical significance.

\section{RESULTS}

We included 599 males and 716 females from 4 to 90 years of age in the study (mean, 52 versus 51 years; $P=.259$ ). Among the 1315 included cases, most CTs were performed for sinusitis or upper respiratory infection (1112 cases, $84.6 \%$ ) (Table 1).

Of 1315 sinus CT cases, $553(42.1 \%)$

\section{MATERIALS AND METHODS \\ Patients}

The study was approved by the institutional review board at Lahey Hospital and Medical Center, which waived the requirement for informed consent. A total of 1389 consecutive sinus CTs during an 8-month period and their associated demographics were retrospectively reviewed. Seventy-four cases were excluded from the study because the most anterior portion of face was not imaged on coronal sequences or there was excessive image degradation due to motion or dental artifacts.

\section{CT Technique}

Direct coronal, nonhelical, sinus CT images were obtained on all patients. Bone and soft-tissue algorithms, display field of view of 15 $\mathrm{cm}$ at a $520 \times 520$ matrix, provided an in-plane resolution of 0.29 $\mathrm{mm}$ (approximately $2 \mathrm{lp} / \mathrm{mm}$ ). Section thickness was $3 \mathrm{~mm}$. In addition, as a function of the deployed CT imaging device, nonhelical 1.5 -mm-thick axial or axial multisection $0.6-\mathrm{mm}$ volumetric CT techniques were used with both bone and soft-tissue algorithms.

\section{Imaging Analysis}

The number of hypodermal radiopaque lesions with Hounsfield units of $>150$ were counted (G.A.F.), and their distribution was recorded for each sinus CT. Validation of positive case numbers was subsequently performed (D.K.).

\section{Statistical Analysis}

To investigate the qualitative association between the subject's age/sex and the presence of facial calcified nodules, we performed logistic regression analysis by using the presence of facial calcified nodules as a binary dependent variable. To investigate the quan- patients had hypodermal calcifications. Most were punctate, $\leq 1$ $\mathrm{mm}$. In some cases, occasional 3- to 4-mm flocculent calcific foci were encountered (Fig 1D). Facial involvement, particularly when extensive, was conspicuously symmetric. The frontal hypodermis was the most frequent distribution of facial calcified nodules ( 480 cases, $86.8 \%$ ), followed by the maxillary hypodermis ( 255 cases, $46.1 \%)$. One hundred ninety-five cases $(35.3 \%)$ demonstrated calcifications in both the frontal and maxillary hypodermis. A few cases demonstrated calcifications in the temporal $(7,1.3 \%)$, mandibular (4, 0.01\%), and periorbital hypodermal tissue (2, 0.01\%). Table 2 summarizes the distribution of calcified nodules by location.

\section{Age and Facial Calcified Nodules}

To investigate the possible association between the presence of facial calcified nodules and the subject's age, we dichotomized the study population into 2 groups with and without facial calcifications. Logistic regression analysis demonstrated that increased age of the subject was strongly associated with the presence of facial calcified nodules (odds ratio $=1.02, P<.001$ ).

For the association between the subject's age and the severity of facial calcified nodules, we used the number of calcified nodules as a surrogate for the severity of disease. At first, linear regression was performed on 553 patients with facial calcified nodules. It demonstrated no statistically significant, quantitative, linear relationship between the subject's age and the number of facial calcified nodules $[b=0.13, F(1,551)=2.79, P=.095]$ with $R^{2}=0.01$ (Fig 2).

Although there is a nonlinear relationship, the severity of facial calcified nodules was also age-dependent. In an effort to investi- 
Table 1: Study population and the distribution of $\mathrm{CT}$ indications ${ }^{\mathrm{a}}$

\begin{tabular}{lcc}
\hline \multicolumn{1}{c}{ Indications } & Cases & $\%$ \\
\hline Chronic sinusitis & 823 & $62.6 \%$ \\
Upper respiratory infection/rhinitis & 142 & $10.8 \%$ \\
Sinus pain/pressure & 76 & $5.8 \%$ \\
Polyposis/mucocele & 35 & $2.7 \%$ \\
Fungal infection & 11 & $0.8 \%$ \\
Allergy & 25 & $1.9 \%$ \\
Asthma & 8 & $0.6 \%$ \\
Headache & 115 & $8.8 \%$ \\
Obstructive sleep apnea & 6 & $0.5 \%$ \\
Mass/cyst & 9 & $0.7 \%$ \\
Head and neck cancer & 8 & $0.6 \%$ \\
Pituitary mass & 6 & $0.5 \%$ \\
Wegener granulomatosis & 4 & $0.3 \%$ \\
Epistaxis & 12 & $0.9 \%$ \\
CSF leak & 6 & $0.5 \%$ \\
Trauma & 3 & $0.2 \%$ \\
Facial infection & 2 & $0.2 \%$ \\
Facial weakness & 3 & $0.2 \%$ \\
Anosmia & 5 & $0.4 \%$ \\
Vertigo/hearing loss & 8 & $0.6 \%$ \\
Otalgia & 1 & $0.1 \%$ \\
Acromegaly & 1 & $0.1 \%$ \\
Unspecified & 6 & $0.5 \%$ \\
Total & 1315 & $100 \%$ \\
\hline The & &
\end{tabular}

${ }^{a}$ The first 6 indications relate to sinusitis or upper respiratory symptoms, accounting for the majority of indications (1112 cases, $84.6 \%$; 1315 sinus CT cases; male, 599, 45.6\%; female, $716,54.4 \%$.

Table 2: Distribution of facial calcified nodules by location

\begin{tabular}{lrcc}
\hline \multicolumn{1}{c}{$\begin{array}{c}\text { Nodule } \\
\text { Distribution }\end{array}$} & All & Male & Female \\
\hline None & 762 & 347 & 415 \\
Frontal & 285 & 126 & 159 \\
Frontal-maxillary & 195 & 87 & 108 \\
Maxillary & 60 & 31 & 29 \\
Temporal & 7 & 3 & 4 \\
Mandibular/buccal & 4 & 4 & 0 \\
Orbital & 2 & 1 & 1 \\
Subtotal & 1315 & 599 & 716 \\
\hline
\end{tabular}

gate any nonlinear effect of age decade and sex of the subjects on the mean number of facial calcified nodules, the Kruskal-Wallis test was performed, revealing a statistically significant difference of the median number of facial calcified nodules among age decade groups $[\mathrm{H}(8)=27.46, P=.001]$. Post hoc comparison analysis between each age decade group with the Dunn test revealed a significantly higher median number of facial calcified nodules in the sixth and seventh decades compared with the second, third, and fourth decades (Fig 3). Additionally, subjects from the fifth, eighth, and ninth decades also had a significantly higher median number of facial calcified nodules than those in the second and third decades (Fig 3).

\section{Sex and Facial Calcified Nodules}

In our study population, 252 males of $599(42.1 \%)$ and 301 of 716 females $(42.0 \%)$ had facial calcified nodules. There was no statistically significant difference between the prevalence of facial calcified nodules between male and female groups $(P=.971)$. By logistic regression, neither male nor female sex was shown to increase the odds of having calcified nodules (odds ratio $=1.02, P=$ .826). Similarly, the Kruskal-Wallis test did not reveal any signif-

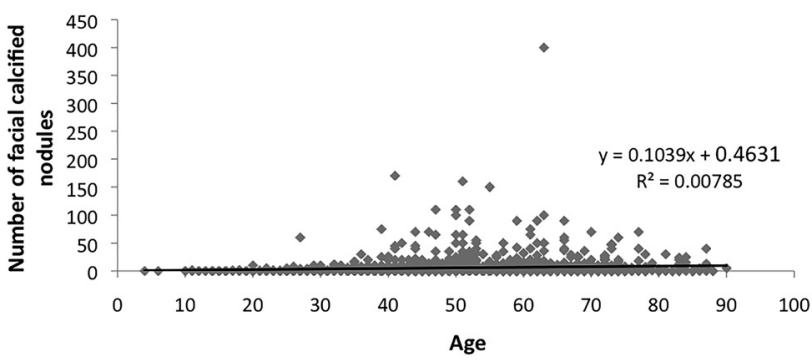

FIG 2. A scatterplot and linear regression between age and the number of facial calcified nodules among 553 patients with positive facial calcified nodules. No significant linear quantitative relationship was observed.
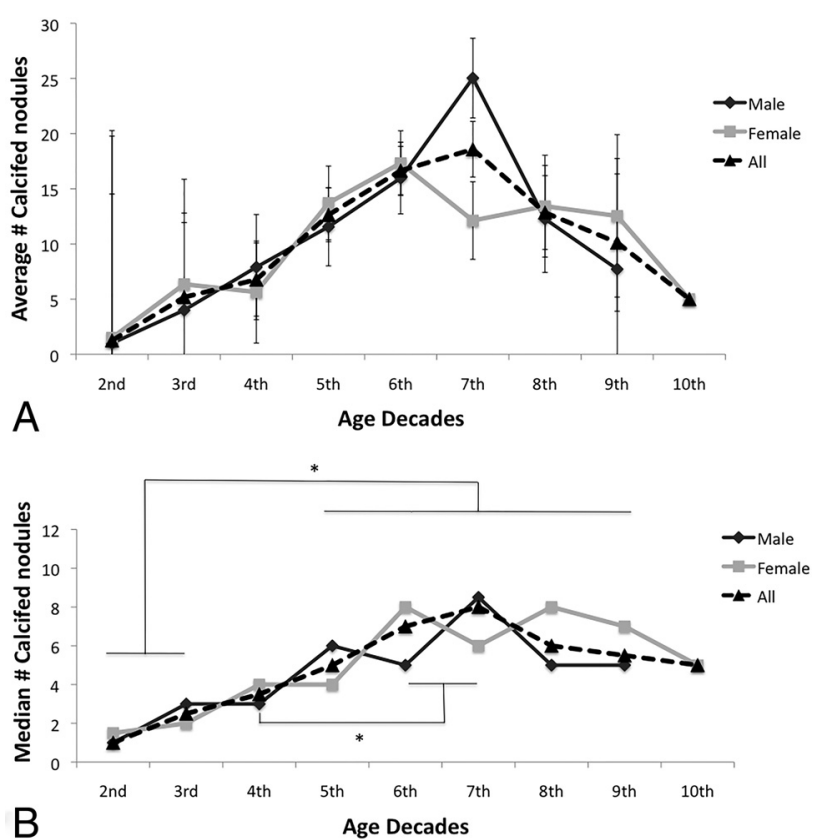

FIG 3. $A$, The average number of facial calcified nodules in each age group by decade. $B$, The median number of facial calcified nodules in each age group by decade. The Kruskal-Wallis test revealed a statistically significant difference in the median number of nodules among different age-decade groups. Post hoc analysis between each age group in the overall population, including both males and females, revealed a significantly higher (asterisk indicates $P<.05$ ) median number of nodules in the fifth-ninth decades compared with the second and third decades. An additional statistically significant difference was observed between the sixth and seventh decades and the fourth decade.

icant effect of the subject's sex on the median number of nodules $[\mathrm{H}(1)=0.032, P=.857]$ among 553 patients with nodules.

\section{DISCUSSION}

Our large-scale, retrospective review of 1315 sinus CT examinations revealed that facial calcified nodules, observed in routine head and face CT imaging, are a common, benign, age-related finding. The prevalence of facial calcified nodules in our study group was $42.1 \%$, indicating that these nodules are a very common finding, compared with $2.3 \%$ in the most recent literature. ${ }^{13}$ The distribution of these facial nodules is symmetric and predominantly involves the frontal and maxillary hypodermal tissue (540/ 553 subjects, $97.6 \%$ ).

Although there was no linear association between the age and 


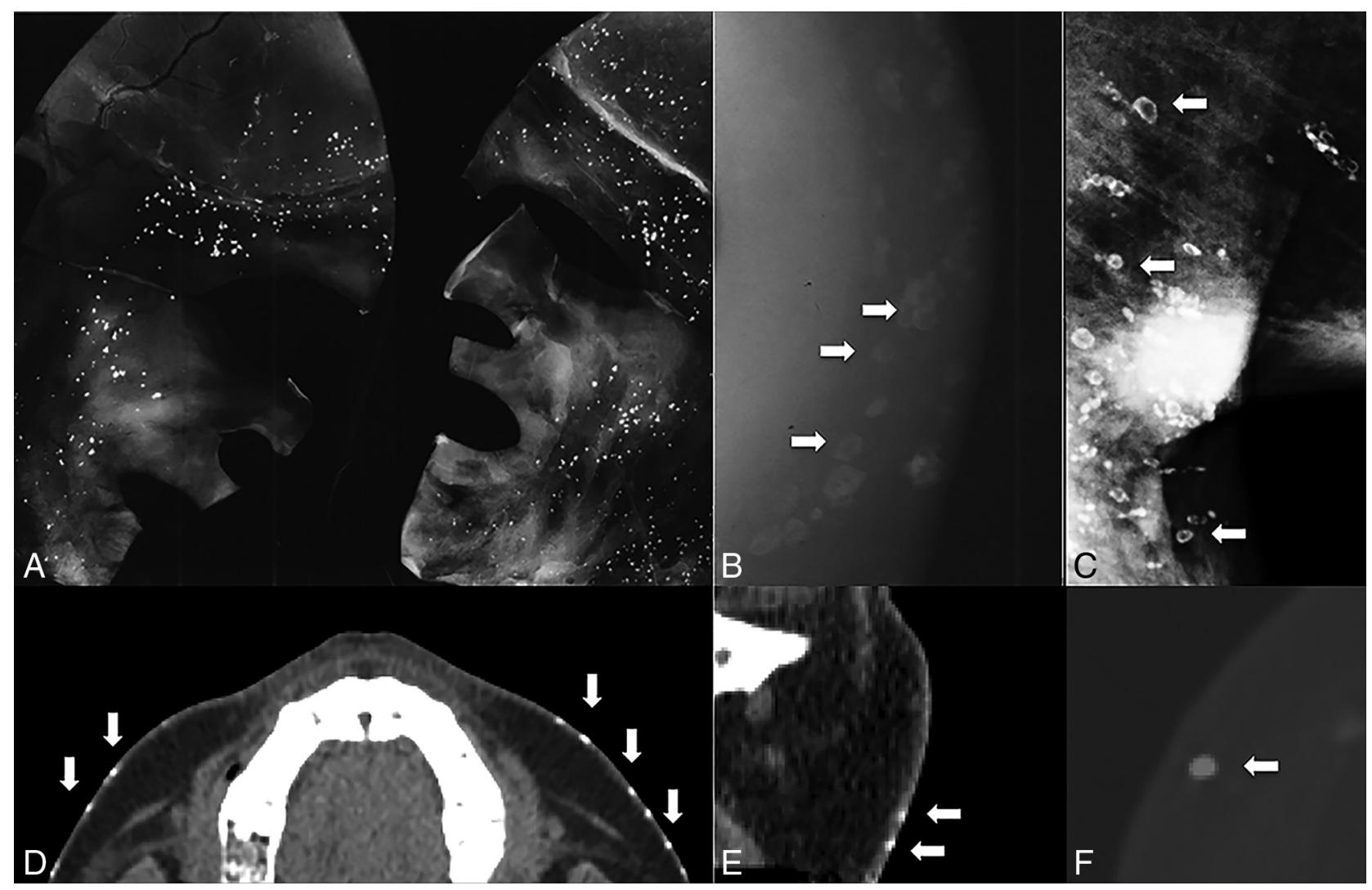

FIG 4. Radiographic examination of cadaveric facial skin specimen $(A)$ reveals multiple calcified nodules. A magnified view of inflated buccal skin on an asymptomatic living patient $(B)$ demonstrates multiple calcified nodules with central lucency. Benign breast skin calcification detected in a routine screening mammogram in a middle-aged female subject $(C)$ demonstrates classic imaging findings of central lucency, which resemble the radiographic appearance of facial calcified nodules in (B). Axial $(D)$ and coronal $(E) C T$ images of the buccal area of a patient demonstrate facial calcified nodules. A magnified axial CT image of a relatively large 5-mm maxillary hypodermal calcified nodule $(F)$ demonstrates a subtle central lucency. $A$ and $B$, Images adapted from Shigehara et al ${ }^{16}$ and obtained from Dr Shigehara with permission.

the severity of facial calcified nodules, our study revealed a strong association between the age and the likelihood of having facial calcified nodules and their severity, supporting the age dependency of these nodules (Fig 3). In contrast to some prior studies, ${ }^{2,14,15}$ we observed no female or male sex predilection for these calcified nodules or their severity.

In 1998, Shigehara et $\mathrm{al}^{16}$ reported a cadaveric case series of 33 individuals. They analyzed facial skin specimens from 19 males and 14 females, with ages ranging from 19 to 85 years. The cause of death for these cadavers was not related to any disease predisposing to secondary osteoma or calcinosis cutis. None of the cadavers displayed obvious signs of changes in the skin (1 exception was fatal burns without involving the face). Radiographic examination of these specimens demonstrated hypodermal, subcutaneous calcified nodules in all 33 subjects (Fig $4 A,-B$ ). They reported that the most frequently involved facial regions were the forehead (29 of $33,87.9 \%$ ) and zygomatic region of the cheek (30 of 33, 90.9\%), similar to the observation in our larger scale study (Table 2). Pathologic and elemental analysis of these nodules revealed a benign, mature bone-like morphology and characteristic and hydroxyapatite-like elemental composition (Fig 5). These nodules demonstrated a concentric, multilamellated, peripheral osteoid, and central adipose medulla, which correlated well with benign, normal bone formation and appeared as well-circumscribed cal- cified nodules with central lucency in radiographs (Fig 4B). In addition, by obtaining dental radiographs, Shigehara et al detected calcifications in $28 \%$ of 158 living subjects and $36 \%$ of subjects older than 40 years of age. They recognized the potential for underestimation due to the limited FOV of dental radiographic techniques.

The radiographic appearance resembles the classic description of benign breast skin calcification, which is believed to be secondary to sebaceous inspissations or low-grade infection. ${ }^{17}$ Routinely encountered breast skin calcification was also described as a "calcified nodule with central lucency" ${ }^{17}$ and appears similar to facial calcified nodules identified in cadaveric specimens (Fig 4C). While the demonstration of central lucency in most facial calcified nodules by CT compared with mammography is limited due to their very small size relative to the section thickness (Fig $4 D$, $-E)$, the appearance of some larger nodules was suggestive of a subtle central lucency (Fig $4 F$ ).

In conclusion, combined evidence from our large-scale sinus CT dataset of 1315 cases with previously reported dental radiographic examinations of 158 patients and a cadaveric study of 33 patients supports our hypothesis that the commonly encountered facial calcified nodules on routine head and neck CT examinations are manifestations of primary miliary osteoma cutis as de- 

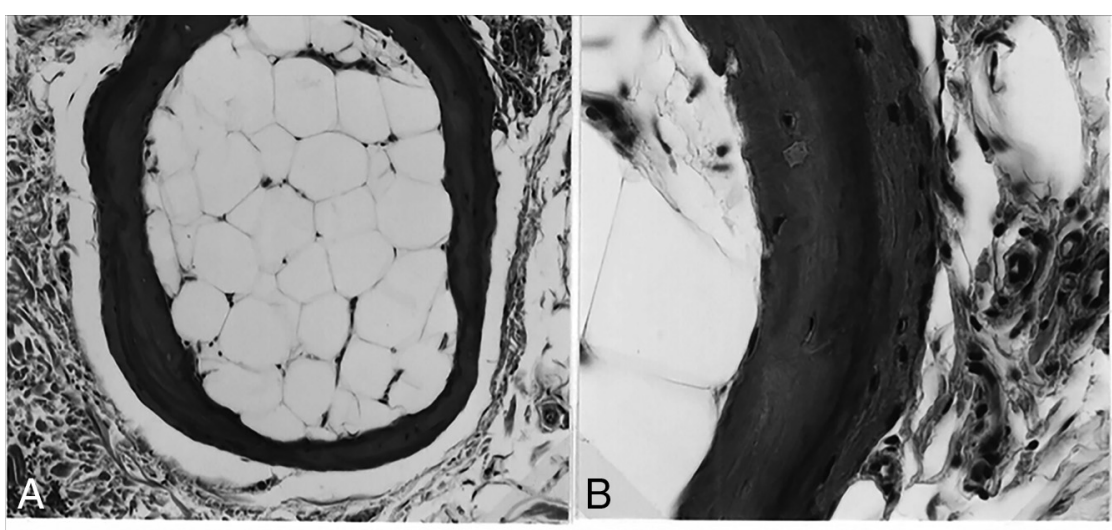

MMO
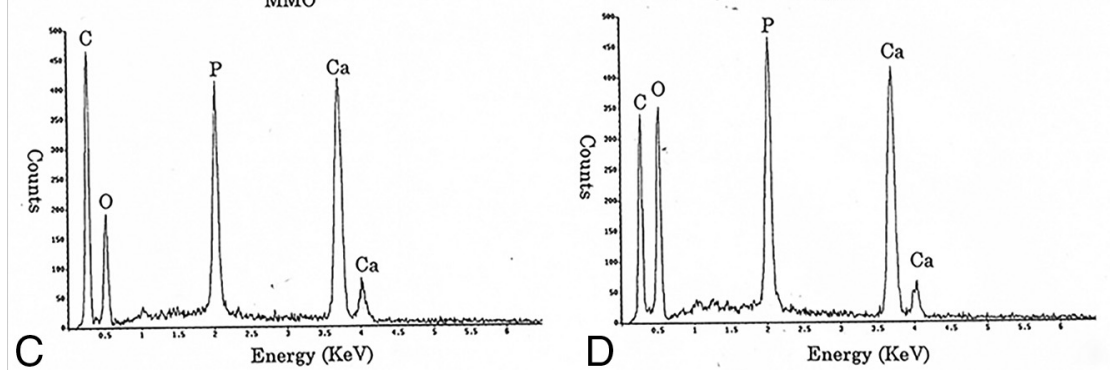

FIG 5. A, Pathologic examination of facial calcified nodules from a cadaveric specimen demonstrates mature bone-like characteristics, including a concentric, multilamellated, osteoid cortex and a central adipose medulla. $B$, A magnified view of a portion of $A$. $C$ and $D$, Elemental composition and peak ratios of a cadaveric facial nodule $(C)$ and the clavicle $(D)$ are similar to each other and well-correlated with the composition of hydroxyapatite. Images are adapted from Shigehara et $\mathrm{al}^{16}$ and obtained with permission from Dr Shigehara. MMO indicates multiple miliary osteoma.

scribed in dermatologic and plastic surgery literature, yet overlooked in the radiologic literature. The discrepancy between previous reports regarding the epidemiology of the disease is likely due to its benign and asymptomatic nature. It is possible that most cases have been overlooked until they are sufficiently advanced or extensive to require dermatologic or surgical attention/intervention. This possibility may also explain the higher incidence of reported cases in females who are more attuned to facial cosmetics than males.

Due to the small, millimetric size of these nodules, it is technically challenging to identify and characterize them (Fig $4 D-F$ ). The FOV in many dental series was restricted to the mandible and maxillary jaw architecture without including the remaining face. Because the forehead was most frequently involved in our series, its exclusion also, in part, accounts for the discrepancy in prevalence from previous reports. Shigehara et al $(1998)^{16}$ observed that "the condition was detected in $28 \%(44 / 158)$ of the living subjects by clinical dental radiographic examination ( $36 \%$ over age 40)."

The major limitation of our study arises from its retrospective design. Although the large number of subjects may have overcome potential confounders, we did not obtain prospective data such as dermatologic history or clinical evaluation. Correlative full-thickness dermatopathology on asymptomatic patients was clearly not possible; thus, we relied on our coauthors' prior large postmortem case series. Some studies suggested the history of severe adolescent acne as a potential precursor of miliary osteoma cutis, ${ }^{4,710,12}$ but this was not formally evaluated in our retrospective, informed-consent-waived study. In addition, because benign breast skin calcification is thought to be secondary to sebaceous inspissations and low-grade infection, one can only speculate that the idiopathic, primary formation of these nodules may be secondary to subclinical or low-grade inflammatory, infectious, traumatic, or environmental insults. The age dependency of facial calcified nodules may, thereby, be explained by longer potential exposure time to any of the above insults than their younger counterparts. In our study group, a small number of subjects demonstrated $>100$ facial calcified nodules (Fig 1C), while the average number of these nodules in a subject was 14 (Figs 2 and 3), raising the possibility of additional disease-exacerbating factors for these individuals with unusually higher numbers of calcified nodules. A prospective, cohort study with multivariable logistic regression could be designed and performed to delineate any of these potential inciting clinical factors in the future.

\section{CONCLUSIONS}

Dermal calcified nodules, observed in routine head and face CT imaging, are common, benign, age-related findings, which have been largely overlooked in the radiology literature. These are a manifestation of primary miliary osteoma cutis.

\section{REFERENCES}

1. Wilckens M. Ueber die Verknöcherung und Verkalkung der Haut und die s. g. Hautsteine. Göttingen: Gottingen University Press; 1858

2. Fazeli P, Harvell J, Jacobs MB. Osteoma cutis (cutaneous ossification). West J Med 1999;171:243-45 Medline

3. Reiter N, El-Shabrawi L, Leinweber B, et al. Calcinosis cutis, part I: diagnostic pathway. J Am Acad Dermatol 2011;65:1-12; quiz 13-14 CrossRef Medline

4. Gfesser M, Worret WI, Hein R, et al. Multiple primary osteoma cutis. Arch Dermatol 1998;134:641-43 CrossRef Medline

5. Walsh JS, Fairley JA. Calcifying disorders of the skin. J Am Acad Dermatol 1995;33(5 pt 1):693-706; quiz 707-10 Medline

6. Duarte IG. Multiple injuries of osteoma skin in the face: therapeutical least invasive in patients with acne sequela: case report. An Bras Dermatol 2010;85:695-98 Medline

7. Jeong KH, Lew BL, Sim WY. Osteoma cutis as the presenting feature of Albright hereditary osteodystrophy associated with pseudopseudohypoparathyroidism. Ann Dermatol 2009;21:154-58 CrossRef Medline

8. Boschert MT, Puckett CL. Osteoma cutis of the hand. Plast Reconstr Surg 2000;105:1017-18 CrossRef Medline

9. O’Donnell TF Jr, Geller SA. Primary osteoma cutis. Arch Dermatol 1971;104:325-26 Medline

10. Myllylä RM, Haapasaari KM, Palatsi R, et al. Multiple miliary osteoma cutis is a distinct disease entity: four case reports and review of the literature. Br J Dermatol 2011;164:544-52 CrossRef Medline

11. Cottoni F, Dell' Orbo C, Quacci D, et al. Primary osteoma cutis: clinical, morphological, and ultrastructural study. Am J Dermatopathol 1993;15:77-81 CrossRef Medline

AJNR Am J Neuroradiol 38:789-94 Apr 2017 www.ajnr.org 
12. Bouraoui S, Mlika M, Kort R, et al. Miliary osteoma cutis of the face. J Dermatol Case Rep 2011;5:77-81 CrossRef Medline

13. Safi $Y$, Valizadeh $S$, Vasegh $S$, et al. Prevalence of osteoma cutis in the maxillofacial region and classification of its radiographic pattern in cone beam CT. Dermatol Online J 2016;22 Medline

14. Boneschi V, Alessi E, Brambilla L. Multiple miliary osteomas of the face. Am J Dermatopathol 1993;15:268-71 Medline
15. Bowman PH, Lesher JL Jr. Primary multiple miliary osteoma cutis and exogenous ochronosis. Cutis 2001;68:103-06 Medline

16. Shigehara $\mathrm{H}$, Honda $\mathrm{Y}$, Kishi $\mathrm{K}$, et al. Radiographic and morphologic studies of multiple miliary osteomas of cadaver skin. Oral Surg Oral Med Oral Pathol Oral Radiol Endod 1998;86:121-25 CrossRef Medline

17. Kopans DB, Meyer JE, Homer MJ, et al. Dermal deposits mistaken for breast calcifications. Radiology 1983;149:592-94 CrossRef Medline 